\title{
Estudio de interacciones en clase de matemáticas: un caso con futuros profesores de matemáticas
}

\section{A Study of Interactions in the Mathematics Classroom: A Case with Pre-service Mathematics Teachers}

\author{
Daniela Pagés ${ }^{1}$ \\ Mónica Olave ${ }^{2}$ \\ Javier Lezama ${ }^{3}$
}

\begin{abstract}
Resumen. En este artículo se presenta el reporte parcial de una investigación en el campo de la Formación del Profesor de Matemáticas donde participaron estudiantes de profesorado de matemáticas de Uruguay. El objetivo fue determinar qué patrones de interacción predominaban en las clases de los futuros profesores participantes, entre los patrones descritos por la Aproximación Interaccionista en Educación Matemática. Para ello se observaron y video grabaron clases de tres estudiantes de profesorado de matemáticas, en el último año de la práctica docente durante su formación, atendiendo a las interacciones sociales que llevan con sus alumnos. En este artículo analizamos episodios de clases de dos de los tres futuros profesores, que fueron seleccionados atendiendo al patrón que configuraban en sus interacciones, y a la relación de dicho patrón con el conocimiento tratado y el momento de la clase en que se configuró. Hemos encontrado evidencia que nos permite concluir que la institucionalización de conceptos o propiedades en la clase puede conducir a la configuración
\end{abstract}

Fecha de recepción: 2 de mayo de 2017. Fecha de aceptación: 22 de febrero de 2018.

${ }^{1}$ Consejo de Formación en Educación de Uruguay (CFE). danielapages@gmail.com

${ }^{2}$ Consejo de Formación en Educación de Uruguay (CFE). monicaolave23@gmail.com

${ }^{3}$ Centro de Investigación en Ciencia Aplicada y Tecnología Avanzada (CICATA), Instituto Politécnico Nacional de México. jlezamaipn@gmail.com 
de los patrones extractivo o de embudo. Proponemos herramientas para analizar estas cuestiones en la formación de profesores de matemáticas.

Palabras clave: interacción social; formación de profesores de matemáticas; significados; patrones de interacción; matemática educativa.

\begin{abstract}
We present a partial research report in the field of Mathematics Teacher Education, in which three uruguayan mathematics pre-service teachers participated. The study aimed to determine which interaction patterns prevailed in their classes, between those described by the Interactionist Approach in Mathematics Teacher Education. Three pre-service mathematics teachers' classes were observed and videotaped. They were developed in the last year of their teaching practice. This article focuses on the analysis of some episodes from two of the three students involved in the study. These episodes were selected in attention to the interaction pattern they configured, as well as the knowledge to be treated and the moment of the class in which they were configured. We concluded that pre-service teachers configured these patterns when they intended to evoke or institutionalize a mathematics statement. We propose the social interaction analysis as a tool to consider these issues in mathematics teacher education.
\end{abstract}

Keywords: social interaction; mathematics teacher education; meanings; interaction patterns; Mathematics education.

\title{
1. INTRODUCCIÓN
}

En este trabajo presentamos parte de los resultados de una investigación realizada con futuros profesores de matemáticas de Uruguay. Esta investigación fue motivada por las dificultades observadas en los estudiantes cuando desempeñan el rol de profesores en la práctica docente del último curso de su formación inicial. La carrera de profesorado de matemáticas en Uruguay consta de cuatro años de cursos, y se estructura en base a tres dimensiones: las ciencias de la educación (Pedagogía, Sociología, Historia de la Educación, entre otras); las asignaturas específicas de matemáticas (Cálculo diferencial e integral, Geometría, Álgebra Lineal, Topología, etcétera) y la Unidad Didáctica - Práctica Docente. Esta última se compone de tres cursos centrados en el cuerpo de saberes de la 
Matemática Educativa, con práctica docente, llamados cursos de Didáctica. En los dos primeros cursos de Didáctica (Didáctica I y II) los Estudiantes de Profesorado de Matemática (en adelante EPM) concurren a un centro educativo para observar las clases de un profesor, y dictan algunas clases bajo la supervisión del docente de Didáctica. En el último año (Didáctica III) los EPM tienen un curso de matemáticas de enseñanza secundaria (12-15 años) totalmente a su cargo. Desde nuestro rol de profesores de Didáctica III, en oportunidad de las visitas a clases de los EPM, hemos observado ciertas dificultades que presentan en relación con los aportes de la Matemática Educativa que estudian en la parte teórica del curso. Estas dificultades se vinculan con las formas de presentar el conocimiento, las actividades que los EPM proponen a sus alumnos, el tratamiento de estas actividades en la clase. Por ejemplo, los roles que los EPM asumen como docentes y los roles que promueven en los estudiantes, la atención a las intervenciones de los alumnos, la conexión de las ideas matemáticas, la promoción del desarrollo de procesos de pensamiento matemático y meta matemático. Estas observaciones nos motivaron a investigar el escenario de la práctica docente de los EPM en la asignatura Didáctica III.

La investigación, de la que reportamos algunos resultados parciales, tomó como marco de referencia la Aproximación Interaccionista en Educación Matemática (adaptada del Interaccionismo Simbólico por Bauersfeld, Krummheuer y Voigt, 1988, citados por Godino y Llinares, 2000). A partir de los principales patrones de interacción que esta aproximación describe, surgidos de estudios efectuados en clases de matemáticas, formulamos la siguiente pregunta de investigación: ¿Qué patrón de interacción predomina en las clases de cada EPM? En este artículo tomamos un aspecto particular de la investigación, enfocado en la descripción de algunos episodios de clases de dos de los EPM participantes. Esto se debe, como hemos dicho, a que nos interesa mostrar la relación entre el patrón de interacción que configuran y el momento de la clase en que se produce, los cuales están vinculados con la institucionalización de los conceptos, y la formulación matemática de una definición o un teorema, respectivamente, para cada caso considerado.

\section{ANTECEDENTES}

A continuación reportamos algunos trabajos relacionados con la temática que abordamos, por sus objetivos o las conclusiones a las que llegan. Esto nos 
brindará un panorama general de lo que se ha investigado en Matemática Educativa en torno a este tema, y nos permite ubicar nuestro proyecto en relación con las investigaciones reportadas, así como destacar los aportes que pretendemos realizar en el campo.

En Olave (2013) encontramos datos aportados por la Sala de Didáctica de la Matemática del Departamento de Matemática de Formación Docente, los cuales señalan que los EPM, en el desarrollo de sus prácticas docentes, presentan a sus alumnos una matemática consistente en un conjunto de proposiciones y sus demostraciones, enunciadas por los EPM, y en las que los alumnos no tienen participación activa.

Blanco (1996) describe y analiza dificultades que los estudiantes de profesorado manifiestan en sus prácticas docentes, señalando que se sitúan más en la perspectiva de alumnos que de futuros docentes en práctica. Reflejan más, en la práctica docente, sus vivencias estudiantiles que lo estudiado en los cursos de formación de profesores.

Even y Markovitz (1991, citados en Ponte y Chapman, 2006) estudiaron el Conocimiento Pedagógico del Contenido en docentes de Secundaria, analizando sus respuestas a las preguntas, puntualizaciones e hipótesis de sus alumnos sobre el tema funciones. Concluyeron que los profesores no siempre son conscientes de las dificultades de sus alumnos, algunos ignoran sus formas de pensamiento, y muchas veces solo los evalúan expresando correcto o incorrecto.

Consideramos que los tres aportes mencionados tienen vinculación entre sí. En efecto, la presentación de las matemáticas como un conjunto de proposiciones ya hechas, establecidas por el aspirante a docente sin intervención de los estudiantes, puede reflejar las concepciones de las matemáticas, su enseñanza y aprendizaje, construidas en la etapa estudiantil del futuro profesor, lo que muestra una dificultad para situarse en el rol de profesor. Desde este rol el futuro docente debería tomar en consideración el pensamiento matemático de sus alumnos, las dificultades que pueden tener, los errores que cometen.

En un escenario argentino, de similares características al uruguayo en cuanto a la formación docente y particularmente a las prácticas, Homilka (2008) analiza las prácticas de estudiantes de profesorado en oportunidad de cursar la Residencia (primera experiencia de práctica docente), en la cual se espera que los futuros docentes integren los conocimientos de matemáticas, de la formación pedagógica y los cursos de Didáctica. La autora señala que los futuros profesores tienen dificultades para estructurar las clases, definir objetivos de aprendizaje, elegir el material didáctico. Establece también que los estudiantes de profesorado 
reproducen prácticas de sus propios profesores, ya sea que las tomen de las recibidas como alumnos, o que adopten prácticas más propias del nivel superior que de la enseñanza media.

Las investigaciones señaladas indican que la problemática que estudiamos no es exclusiva de algunos futuros docentes ni está limitada al contexto de nuestro país, sino que es una dificultad presente de forma más amplia en la formación de profesores de matemáticas. Pensamos que la investigación que reportamos proporciona evidencias que permiten establecer en qué momentos de la clase se presentan las dificultades reportadas, y cómo el EPM las resuelve. También se presentan explicaciones en términos de los patrones de interacción que se configuran y la negociación de significados que estos permiten llevar adelante.

\section{EL MARCO TEÓRICO DE LA INVESTIGACIÓN}

\subsection{LA CLASE DE MATEMÁTICAS COMO OBJETO DE ANÁLISIS - ¿QUÉ DIMENSIONES DE LA CLASE ESTUDIAR?}

Entre las múltiples dimensiones de la clase de matemáticas (su planificación, el diseño de las tareas, la evaluación, las interacciones del docente con los estudiantes y de estos entre sí, el conocimiento matemático del profesor, sus creencias y concepciones), en esta investigación optamos por el análisis de las interacciones que los EPM participantes desarrollaban en las clases observadas. Lo hicimos desde la premisa de que en las interacciones de la clase se pone en acción lo que el docente planificó y pensó en relación con la misma. Consideramos que así nos sería posible observar aquellos elementos que nos permitirían analizar la problemática considerada: el rol, tanto del EPM como profesor como el de los estudiantes, las normas que se establecen en la clase, el valor que el EPM da a las intervenciones de los alumnos y si las ve como manifestación de lo que los estudiantes piensan, o como partes de una respuesta esperada por el EPM, entre otros aspectos que se desarrollan más adelante.

En Radford (2010: 102) encontramos una reseña de la consideración de la interacción en la clase de matemáticas como constructo dentro de la Matemática Educativa, desde su origen. Este autor señala que el concepto de interacción apareció como objeto de estudio en la disciplina luego de un cambio drástico en la conceptualización de la enseñanza y el aprendizaje. Cita su origen en el 
desarrollo del marco del Interaccionismo Social (que aquí llamamos Aproximación Interaccionista en Educación Matemática).

Junto con la interacción como materia de estudio aparece el concepto de negociación de significado, ya que se pasa a considerar la construcción social del mismo a través de la interacción humana. De este modo, las ideas de interacción social y negociación de significados están íntimamente ligadas, ya que la consideración de la primera supone, en el proceso comunicativo de la clase, la interpretación de las intervenciones de cada participante por parte de los demás y, a partir de ella, la necesidad de negociar un significado entre los múltiples atribuidos por los distintos participantes a los objetos matemáticos (Radford, 2010).

Consideramos, por tanto, que la elección de las interacciones de la clase como objeto de estudio e investigación resulta pertinente, por la información que nos permite obtener en relación con las dificultades observadas en los EPM durante sus prácticas docentes, vinculadas con los distintos aspectos de la clase. A continuación describimos los elementos principales de esta aproximación.

\subsection{LA APROXIMACIÓN INTERACCIONISTA - LOS PATRONES DE INTERACCIÓN}

La aproximación interaccionista en Educación Matemática está influenciada de forma particular por el Interaccionismo Simbólico (Blumer, 1969 y Mead, 1934, citados por Voigt, 1995) y por la etnometodología (Garfinkel, 1967; Mehan, 1979, citados por Voigt, 1995).

Bauersfeld et al. (1985) adaptaron los conceptos sociológicos del Interaccionismo Simbólico a los de la enseñanza y el aprendizaje de las matemáticas, dando surgimiento así a la Aproximación Interaccionista en Educación Matemática. Esta considera que la evolución del conocimiento matemático, al igual que sus fuentes, tienen una gran influencia sociocultural (Sierpinska y Lerman, 1996). Las matemáticas son consideradas resultado de los procesos sociales (Lakatos, 1976; Wittgenstein, 1967, citados por Voigt, 1995) y no como un conjunto de relaciones verdaderas, objetivas e inmutables entre objetos, como lo establecen las teorías platónicas o intuicionistas.

Desde la Aproximación Interaccionista en Educación Matemática se sostiene que, al contrario de lo que se cree popularmente, los objetos de la clase de matemáticas (los conceptos, símbolos, las tareas que se plantean, lo que se dice) no tienen significado único. Cada clase es considerada una micro cultura, y cada participante de ella interpreta estos objetos de acuerdo con sus conocimientos y 
experiencias anteriores, los que constituyen un contexto para la interpretación que hace de ellos. Es decir, estos objetos son ambiguos, lo cual trae consigo la necesidad de negociar los significados que se tomarán por compartidos en la clase. Dicha negociación solo se produce a través de la interacción social, entendida como un conjunto de interpretaciones de las expectativas de los demás y de los significados atribuidos a los objetos, así como un conjunto de acciones determinadas por esas interpretaciones. (Bauersfeld et al., 1985; Voigt, 1995).

Bajo los supuestos recién descritos, el interés del investigador (para el caso de la aproximación en Educación Matemática) se focaliza en cómo se aprenden matemáticas en las interacciones de la clase, para lo que se consideran apropiados los métodos micro-etnográficos. A través de observaciones de clases video grabadas, el observador accede a la información buscada por medio de la interpretación de reconstrucciones (Cicourel, 1973, citado en Bauersfeld et al., 1985). Estas interpretaciones persiguen el objetivo de comprender las acciones como se ven desde la perspectiva de un sujeto, o interpretadas por reglas de interacción.

Bauersfeld et al. (1985) señalan, apoyados en las observaciones de clase realizadas, que la negociación de significado producida en la clase no siempre es la más adecuada para generar riqueza en los conocimientos que se terminan compartiendo. La preocupación del docente por presentar el tema planificado, o llegar a la solución del problema que ha planteado, como forma de cumplir con su tarea, y la interpretación que los estudiantes hacen de las expectativas docentes, van formando rutinas y patrones de trabajo que, si bien ayudan a disminuir la complejidad de la clase, muchas veces se vuelven estereotipadas y terminan siendo una ficción de negociación de significados por la que se llega a la "solución oficial" de la tarea.

Comenzamos por explicar lo que estos autores entienden por patrón de interacción.

Un patrón de interacción es una estructura de interacción cara a cara entre dos o más sujetos, tal que:

- sirve para reconstruir una regularidad específica de interacción focalizada en un tema,

- refiere a acciones concertadas, interpretaciones y mutuas percepciones de al menos dos participantes, y no es la suma de sus acciones individuales,

- la estructura no es explicable por medio de un conjunto de reglas, 
- los participantes en esa estructura la generan de manera inconsciente y sin un propósito estratégico, la constituyen rutinariamente.

Dichos autores describen cuatro patrones de interacción: el extractivo y el de discusión (Voigt, 1985), por un lado, y el de embudo y el de focalización (Wood, 1994) por otro.

En la siguiente tabla presentamos las fases que caracterizan a cada uno de ellos.

Tabla 1. Descripción de los patrones de interacción social por sus fases.

\begin{tabular}{|c|c|c|c|c|}
\hline \multirow{2}{*}{ Fases } & \multicolumn{4}{|c|}{ Patrones de interacción } \\
\hline & Extractivo & Embudo & Discusión & Focalización \\
\hline 1 & $\begin{array}{l}\text { El docente plantea } \\
\text { una pregunta o } \\
\text { actividad y los } \\
\text { estudiantes dan } \\
\text { respuestas, hasta que } \\
\text { el docente toma una } \\
\text { que considera útil } \\
\text { para la solución que } \\
\text { ha pensado para la } \\
\text { cuestión planteada. }\end{array}$ & $\begin{array}{l}\text { El docente plantea } \\
\text { una pregunta o } \\
\text { actividad y los } \\
\text { estudiantes dan } \\
\text { respuestas, pero no } \\
\text { consigue una } \\
\text { respuesta útil. }\end{array}$ & $\begin{array}{l}\text { El docente plantea } \\
\text { una actividad para } \\
\text { que los estudiantes } \\
\text { la realicen, } \\
\text { individualmente o } \\
\text { en grupos. }\end{array}$ & $\begin{array}{l}\text { El docente } \\
\text { plantea una } \\
\text { actividad para } \\
\text { que los } \\
\text { estudiantes la } \\
\text { realicen, } \\
\text { individualmente } \\
\text { o en grupos. }\end{array}$ \\
\hline 2 & $\begin{array}{l}\text { A partir de dicha } \\
\text { respuesta el profesor } \\
\text { guía a los alumnos, } \\
\text { mediante nuevas } \\
\text { preguntas y pistas, a } \\
\text { la solución. }\end{array}$ & $\begin{array}{l}\text { El profesor continúa } \\
\text { preguntando, con } \\
\text { espacio cada vez } \\
\text { menor para la } \\
\text { respuesta, casi como } \\
\text { dejando espacios en } \\
\text { blanco. }\end{array}$ & $\begin{array}{l}\text { Uno o varios } \\
\text { estudiantes } \\
\text { explican y } \\
\text { fundamentan su } \\
\text { solución. El } \\
\text { profesor colabora } \\
\text { con la explicación } \\
\text { del estudiante, a } \\
\text { través de preguntas } \\
\text { adicionales, } \\
\text { sugerencias, } \\
\text { reformulaciones o } \\
\text { juicios, de modo } \\
\text { que surge una } \\
\text { explicación conjunta } \\
\text { que se da por } \\
\text { válida. }\end{array}$ & $\begin{array}{l}\text { Uno o varios } \\
\text { estudiantes } \\
\text { explican y } \\
\text { fundamentan } \\
\text { su solución. }\end{array}$ \\
\hline
\end{tabular}




\begin{tabular}{|c|c|c|c|c|}
\hline 3 & $\begin{array}{l}\text { El profesor resume lo } \\
\text { que han hecho, } \\
\text { vinculando la } \\
\text { pregunta inicial con el } \\
\text { procedimiento } \\
\text { seguido. }\end{array}$ & $\begin{array}{l}\text { Se llega a la } \\
\text { respuesta buscada, } \\
\text { no importando quién } \\
\text { la dice. }\end{array}$ & $\begin{array}{l}\text { El docente } \\
\text { pregunta si hay } \\
\text { otras soluciones, y } \\
\text { si las hay, los } \\
\text { estudiantes que las } \\
\text { han producido las } \\
\text { explican y } \\
\text { fundamentan. }\end{array}$ & $\begin{array}{l}\text { El profesor, } \\
\text { consciente de la } \\
\text { incomprensión } \\
\text { de algunos } \\
\text { estudiantes, } \\
\text { promueve que } \\
\text { formulen } \\
\text { preguntas, y } \\
\text { pide a quien } \\
\text { explicó la } \\
\text { solución, que } \\
\text { las responda. } \\
\text { Busca la } \\
\text { explicación del } \\
\text { propio } \\
\text { estudiante. }\end{array}$ \\
\hline
\end{tabular}

Las fases establecidas en la descripción de cada patrón se establecen desde un punto de vista analítico, pero pueden solaparse entre ellas en la práctica. Los patrones de embudo y de focalización, si bien pueden parecer similares, contienen interacciones que difieren en los significados que se negocian, así como en el rol que el docente asume y el rol que le otorga al estudiante. Wood (1994, 1998) ejemplifica el patrón de embudo con el siguiente episodio:

Maestro: ¿Cuánto es $9+7$, Jim?

Jim: 14.

Maestro: Ok. $7+7$ es igual a 14. (Escribe $7+7=14$ ). 8 más 7 es justo uno más que 14 , es decir__ ? (Subiendo un poco la voz mientras escribe $8+7=$

Jim: 15.

Maestro: Y 9 es uno más que 8. Entonces ¿15 más uno más es ...? (Vuelve a subir un poco la voz al escribir).

Jim: 16.

Maestro: Correcto.

(Wood, 1998: 170-171, traducción de los autores). 
En la configuración del patrón de embudo parece, inicialmente, que el docente toma la respuesta incorrecta del estudiante, e inicia un diálogo en que intenta que el mismo alumno pueda corregirse, desarrollando su pensamiento. Pero el conjunto de preguntas que le va haciendo incluyen solamente lugares en blanco para que dé la respuesta, de modo que puede responder solo "sumando de a uno", siguiendo las indicaciones del maestro, pero el estudiante no está considerando ninguna propiedad de las operaciones por sí mismo. Más bien, quien está considerando cognitivamente estas propiedades es el docente, y el final de la interacción se da con la respuesta esperada. Según Wood (1998), este es un ejemplo de interacción unívoca (Wertsch y Toma, 1995, citado en Wood, 1998), descrita como aquella que enfatiza el aspecto transmisivo de la comunicación.

Wood $(1994,1998)$ también describe el patrón de focalización. La autora parte de un episodio que se inicia con una explicación, dada por un estudiante, de su forma de restar 66 menos 28. Esto forma parte de una tarea en la que los alumnos desarrollan sus propios métodos de cálculo, trabajando en pequeños grupos (en la clase no se enseñaban los algoritmos tradicionales de las operaciones).

El episodio transcurre así:

John: (Está escribiendo en el proyector). Pusimos el 28 debajo del 66. (Mientras habla, escribe 66 menos 28 en un formato vertical). Y nos llevamos ... nosotros ... yo quité ... el 6 y el 8. Y dijimos que había 60 y 20 allí. (Pone su dedo sobre el 60 y luego sobre el 40). Y si restas 20 de 60, te da 40. (Levanta su puño). Y todavía tengo que restar 8. Entonces sacamos ... hay 46 todavía. Si devuelves esos 6 , y quitas ese 6 (señala el 6 en el 46), y eso ... um ... vuelves a 40 y todavía tienes que quitar 2, entonces son 39 (levanta un dedo), entonces 38 (escribe 38). (Wood, 1998: 172-173, traducción de los autores).

(La maestra reconoce que John ha desarrollado un nuevo y exitoso procedimiento para lidiar con la dificultad de la resta, usando abordajes que habían surgido para efectuar sumas. Pero en lugar de explicar nuevamente para la clase, pregunta si tienen alguna pregunta. Un alumno dice que no entiende, y la maestra pide a John que vuelva a explicar. El episodio continúa). 
John: Pusimos el 66 y debajo el 28. Luego quitamos el 6 y el 8 y, si sacas 20 de 60, da 40. Y si vuelves a poner el 6 y el 8, tienes 46. Entonces quitamos, todavía tenemos que quitar ese 8. Entonces quitas ese 6, ahora tienes de nuevo 40 y todavía tienes que quitar 2.

Elisabeth: Pero, pero ¿ipor qué quitaste el 6 y el 8?

John: Era más fácil.

Maestra: (Mirando a la clase y decidiendo que los otros podrían aún no entender lo que hizo), Ok, ¿ipuedes escribir al lado qué fue lo que hiciste? Tal vez nos ayude a verlo. En lugar de 66 menos 28, ¿qué hiciste?

John: A 60 le resto 20 es igual a 40 (escribe 60 menos 40 verticalmente, y mira a la maestra).

Maestra: ¿Puedes escribir lo que obtuviste? (Escribe 40 debajo de 60 menos 20.). OK, ¿qué hiciste luego?

John: Luego puse nuevamente el 6. Entonces es igual a 46. (Escribe +6 cerca del 40). Y todavía

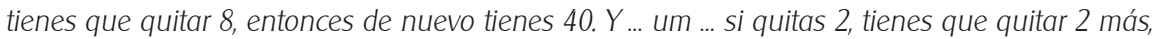
entonces tienes 38 .

Maestra: (a la clase) ¿Tiene sentido? (Pausa). ¿Entienden lo que dijo sobre esta parte? Dijo (va hacia adelante para usar el proyector). Tengo, pongamos este 46 aquí (escribe 46 en la parte superior). Esto es lo que él tiene y dijo "tengo que volver a 40". OK, ¿por qué vuelves a 40?

(Wood, 1998: 173-174, traducción de los autores).

El episodio culmina cuando John explica la razón de los últimos pasos. Al pedir al estudiante que vuelva a explicar, la maestra está permitiéndole que clarifique su pensamiento a los compañeros. Y formula preguntas para mantener el foco de la atención en aquellos aspectos que ha notado que muchos estudiantes no comprendieron. La cuestión directiva de la maestra es un intento de mantener la discusión en torno a los aspectos distintivos de la resolución de John. Este es un patrón diferente del de embudo, en tanto la pregunta se dirige a generar la reflexión sobre una forma de pensamiento. No guía al alumno, sino que le da la responsabilidad de explicar. En relación con los aspectos señalados por Wertsch y Toma (1995), diríamos que el énfasis está puesto en los aspectos dialógicos de la comunicación.

Los patrones no son entendidos como la suma de las acciones de cada participante, sino como un conjunto de interpretaciones y obligaciones mutuas entre ellos. Por esto, a través de la interacción se van generando condicionamientos y expectativas que orientan el desarrollo del propio patrón. Por ejemplo, en la primera fase del patrón extractivo el estudiante tiene la obligación implícita de responder la pregunta del docente $u$ ofrecer soluciones posibles. En la 
segunda fase, en la que el profesor espera llegar a la respuesta esperada, su esfuerzo interpretativo consiste en detectar aquellas respuestas que le pueden ser útiles para ese fin. Los estudiantes tienen el compromiso de ir respondiendo, y no demostrar que no están comprendiendo. En la tercera fase, el docente tiene la obligación de relacionar las ideas de los alumnos con la solución a la que se llegó, explicando el camino hacia ella.

Los patrones también reflejan ciertas creencias sobre la naturaleza de las matemáticas, y de las relaciones entre los estudiantes y el docente. El patrón de embudo deja translucir que las matemáticas que se van a aprender solo descansan en la autoridad del profesor (Wood, 1998). En el patrón de focalización, en cambio, se parte de una forma de trabajo generada por las acciones de los alumnos, y en la que el docente focaliza aquellos aspectos que le parece importante destacar.

Otro de los conceptos importantes de este marco son las normas sociales y sociomatemáticas establecidas en la clase, y que se conectan con preguntas como: ¿quién puede hacer preguntas en la clase?, ¿quién puede responder preguntas?, ¿cuándo una explicación se considera correcta?, ¿quién determina si una resolución es correcta o no?, etc. De las distintas normas que se establezcan, así como de las rutinas y patrones configurados, dependerán las características de la cultura de la clase, y es a través de ellas que esta será creada.

\subsection{REORGANIZACIÓN DE LOS PATRONES PARA EL ESTUDIO REALIZADO}

Hemos encontrado similitudes entre algunos de los patrones descritos por la Aproximación Interaccionista en Educación Matemática, que nos llevan a agruparlos. A continuación explicamos las razones de este agrupamiento.

El patrón de embudo muestra una idea sobre las matemáticas, su enseñanza y su aprendizaje. Los estudiantes no necesitan involucrarse cognitivamente en la actividad para responder correctamente las preguntas, sino que es el docente quien lo hace, y sabe a qué respuesta quiere llegar. Algunas de estas características son comunes con el patrón extractivo.

En el patrón de focalización se establecen formas de trabajo propias del patrón de discusión, como el trabajo de los alumnos y la responsabilidad que el docente les otorga en la explicación y justificación, el objetivo del profesor en cuanto al desarrollo del pensamiento de los estudiantes y la finalidad de las tareas, entre otras. 
Las consideraciones anteriores nos llevaron a agrupar los patrones en dos categorías. En la primera incluimos el patrón extractivo, y como posible fase, el desenlace hacia el patrón de embudo. En la segunda categoría ubicamos el patrón de discusión y, en cierto momento dentro del mismo, el de focalización. A continuación presentamos dos tablas donde se muestran las categorías mencionadas.

Tabla 2. Patrones extractivo-embudo.

\section{Fase 1}

El docente presenta una tarea (pregunta o problema), los estudiantes plantean respuestas, el docente las evalúa preliminarmente (correctas, incorrectas, útiles, etc.). Esto sigue hasta que el profesor encuentra una respuesta útil a sus objetivos.

Fase 2

Desarrollo guiado de la solución definitiva. El docente, a través de pistas, gestos, nuevas preguntas, va guiando las respuestas de los alumnos.

Fase 3

El profesor realiza una evaluación del método empleado y del resultado obtenido, y se reflexiona sobre el contexto. Esta fase no siempre se da.
Patrón de embudo

Los estudiantes no logran responder lo esperado por el docente, entonces este interviene de forma más directa, con preguntas que van reduciendo el campo de acción del estudiante y le van señalando la respuesta esperada.

La diferencia entre la configuración del patrón extractivo y su desenlace al patrón de embudo tiene que ver con que, cuando este último se configura, generalmente ante una respuesta incorrecta, disminuye drásticamente el involucramiento cognitivo del alumno con la resolución de la tarea. Es el docente quien dirige la misma, dejando al estudiante un pequeñísimo lugar para la respuesta esperada. 
Tabla 3. Patrones de discusión- focalización.

Fase 1

El docente propone una tarea, preferentemente para hacer en grupos, pero puede ser individual.

Fase 2

Pide a los estudiantes que expongan lo que hicieron, y lo justifiquen.

Fase 3

Un estudiante (o varios) da su solución, explicando.

Fase 4 (Puede mezclarse con la 3)

El profesor realiza preguntas, comentarios para enfatizar, aclarar o profundizar.

Pregunta por otras resoluciones.

\section{Patrón de focalización}

Las preguntas del docente tienen como objetivo focalizar la atención de los estudiantes en algún aspecto del problema, que es crucial para el significado que quiere promover, o que no han tenido en cuenta en la resolución.

Fase 5

Otros estudiantes explican su solución.

El patrón de discusión se caracteriza, en su Fase 4, por la intervención del docente haciendo preguntas aclaratorias, o agregando comentarios que permitan llegar a una solución conjunta. En cambio, en el de focalización, el profesor apunta más a que los estudiantes clarifiquen su pensamiento al tener que explicitarlo a sus compañeros.

\section{ASPECTOS METODOLÓGICOS}

En la investigación reportada utilizamos la etnografía como metodología. Se analizaron algunos episodios de interacciones de clase para cada uno de los tres EPM que participaron. Estos fueron individualizados, para el análisis de los datos, como EPM1, EPM2 y EPM3 (aquí solo analizamos interacciones del EPM1 y del EPM3). Se visitaron y video grabaron cuatro clases de cada uno de los EPM participantes. Para determinar, luego de las observaciones, el patrón predominante para cada EPM, elaboramos un protocolo de observación de clases (Tabla 4) basado en la comparación de los patrones (con su agrupación explicada en las Tablas 2 y 3) de acuerdo con sus características principales (Wood, 1994; Wood, 1998; Voigt, 1985), así como con las normas sociales y sociomatemáticas de la clase (Stephan y Cobb, 2003): 





En el análisis de cada episodio utilizamos la Tabla 4 para detectar las características de uno u otro de los patrones, tal como fueron agrupados.

\section{ANÁLISIS DE EPISODIOS SELECCIONADOS}

En este apartado presentamos el análisis de dos episodios de las interacciones correspondientes al EPM1 y al EPM3 que ilustran aspectos que nos permitieron determinar qué patrón de interacción predomina en sus clases. Reiteramos el motivo de elegir estos dos participantes: consideramos que la configuración de una forma de interactuar propia del patrón extractivo-embudo, en el caso de ellos, se vincula con el propósito del EPM1 y del EPM3 en esos episodios, en cuanto al tratamiento matemático. Si bien las interacciones tratan diferentes contenidos matemáticos, el objetivo de la pregunta que provoca la interacción es que los estudiantes digan una definición (de raíz de una función) para el caso del EPM1, o enuncien un resultado matemático (teorema de Pitágoras) en la clase del EPM3. Es decir, hay una intención de obtener un resultado disciplinar determinado. Parecería que podría existir una relación entre el propósito de la pregunta que inicia el episodio y la configuración de uno de estos patrones. A continuación se analiza para cada caso el patrón que se configura.

\subsection{EL CASO DEL EPM1}

El EPM1 plantea en el pizarrón la tarea que se transcribe a continuación.

Sea $\mathrm{g}: R \rightarrow R, \mathrm{~g}(\mathrm{x})=2 \mathrm{x}+6$

a) Calcula raíz y ordenada en el origen.

b) Represéntala gráficamente.

c) Mirando el gráfico indica $f(x)<0$ si ............

Comienza pidiendo atención a los alumnos y señalando la expresión analítica, pregunta qué es, esperando que le digan "expresión analítica". Una vez que lo logra, les pregunta si recuerdan la definición de raíz de una función. Luego se revisa cuál era la ordenada en el origen, se determina esta y se representa gráficamente la función. Se revisan conceptos como: crecimiento, decrecimiento, los 
elementos de la expresión analítica de los que dependen. Finalmente, se analiza en discusión de toda la clase, la parte c) de la actividad.

Al inicio de la resolución ocurre la siguiente interacción entre el EPM1 y los estudiantes:

\section{Interacción 1 EPM1.}

\section{EPM1: La raíz era, ¿qué era la raíz?}

E5: La ordenada en el origen.

EPM1: La ordenada en el origen es algo distinto.

(Hablan varios a la vez, no se oye).

E6: La preimagen.

EPM1: La preimagen me gusta un poquito más, pero sola no me dice nada la preimagen.

E6: De cero.

E7: La imagen.

EPM1: La preimagen de cero, está bueno, me gusta. pero ¿qué quiere decir que la raíz es la preimagen de cero?

E8: Es el opuesto.

EPM1: Yo estoy de acuerdo con eso (se refiere a la preimagen de cero).

E9: Le corresponde el cero en la gráfica.

EPM1: Que le corresponde el cero en la gráfica. A ver si alguien me lo puede explicar un poquito mejor. La raíz es la preimagen de cero, estoy de acuerdo. Hay un cero en la vuelta, estoy de acuerdo.

E9: (Lee) Llamamos raíz a la abscisa del punto, al corte de la representación gráfica de la función con el eje $x$.

EPM1: A la abscisa del punto de corte.

E9: Sacamos una flechita (se refiere a la anotación en su cuaderno).

EPM1: Bueno, ahí va. ¿Qué era la raíz? El valor, el valor de x cuyo correspondiente es el cero. Es decir, yo quiero averiguar cuánto vale x para que esa cuenta me dé cero.

Durante la primera parte de la tarea, el EPM1 parece tener interés en que los alumnos recuperen el concepto de raíz de una función como el número cuya imagen en la función es cero y, a partir de esta definición, recuerden que tienen que resolver la ecuación $f(x)=0$ (interpretamos esto por los rechazos sucesivos de conceptos correctos expresados por los estudiantes), tomando otro contexto de representación. Además, porque en el episodio siguiente, que aquí no analizamos, se realiza la tarea de hallar la raíz de la función resolviendo la ecuación $f(x)=0$ ). Al inicio del episodio los alumnos dan respuestas erróneas (por ejemplo "la ordenada en el origen") y el EPM1 no propone discutir el error, sino que 
continúa esforzándose por obtener al menos una parte de la respuesta que espera. En las líneas que siguen, los estudiantes encuentran elementos en el cuaderno y proponen definiciones que son correctas, en diferentes registros de representación, y el EPM1 no parece aceptar ninguna de ellas.

Podemos ver, durante este episodio, el desarrollo de la Fase 1 e inicio de la Fase 2 del patrón que hemos Ilamado extractivo-embudo. El EPM1 realiza una pregunta, y recibe propuestas de respuesta de parte de los alumnos. Las mismas son rechazadas con distintas frases del EPM1, hasta que toma (parcialmente) la respuesta de la línea 14, la enuncia corrigiéndola, dando así inicio a la Fase 2 (que no se analiza en este episodio, en la cual se realiza la resolución guiada de la tarea de hallar la raíz de la función).

En las intervenciones de los estudiantes y en las reacciones del EPM1 ante ellas podemos apreciar las características señaladas en la Tabla 2 para el patrón extractivo-embudo. La pregunta del EPM1 busca una respuesta útil para realizar la tarea. Los estudiantes dan respuestas que divergen de lo que el EPM1 espera (L2: la ordenada en el origen; L5: la preimagen). Estas respuestas son breves e incompletas, y los alumnos las van completando a partir de frases del EPM1 (L6: La preimagen me gusta un poquito más pero sola no me dice nada la preimagen). Hay algunas respuestas que el EPM1 no considera (L8: Ia imagen; L10: es el opuesto).

Además, la tarea se resuelve desde el inicio entre el EPM1 y los alumnos, no habiendo lugar para la búsqueda real de la definición de raíz de una función, ya que cada respuesta es evaluada por el docente en forma inmediata. La intención de las preguntas parece ser que los estudiantes digan la respuesta adecuada para realizar la tarea (hallar la raíz de la función). No hay un fundamento para los rechazos de respuestas correctas (la preimagen de cero, la abscisa del punto del corte de la representación gráfica de la función con el eje x) y, por tanto, no existe esfuerzo cognitivo para los alumnos. Ellos dan respuestas, el EPM1 las rechaza o acepta. Además, los estudiantes no conocen la intención de la pregunta del docente.

A partir del análisis anterior podemos inferir que el desarrollo del episodio analizado coincide con la descripción del patrón extractivo-embudo. La intervención docente aparece a primera vista como una forma de sostener el aprendizaje de los alumnos. Pero esto no sucede finalmente. En efecto, el EPM1 no evalúa las respuestas de manera definitiva, diciendo "está mal", sino que sigue el intercambio e intenta guiar hacia la definición esperada, para este caso. A pesar de que puede parecer que el estudiante está involucrado en una estrategia de pensamiento, 
quien lo está realmente es el EPM1, que conoce la respuesta a la que quiere llegar. Esta forma de interacción disminuye el campo para la actividad significativa, tanto grupal como del alumno.

\subsection{EL CASO DEL EPM3}

El EPM3, en la clase cuyo análisis presentamos a continuación, propone la primera parte de la siguiente actividad como introducción al teorema de Pitágoras.

1) La siguiente figura fue creada por H. E. Dudeney (1857-1930). Recorta de allí las piezas 1, 2 ,

3,4, y 5 , y forma con ellas el cuadrado de lado $\mathrm{c}$.

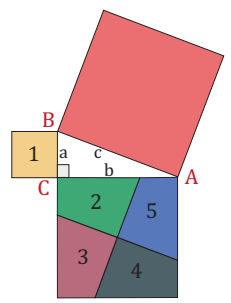

a) Clasifica el triángulo $A B C$ según sus ángulos.

b) ¿Qué relación puedes establecer entre las áreas de los cuadrados construidos sobre los catetos del triángulo $A B C$ y el área del cuadrado construido sobre su hipotenusa?

c) Expresa el área de cada cuadrado en función de la medida de su lado y plantea una fórmula que relacione las tres áreas.

2) La relación que estableciste en la actividad anterior es conocida con el nombre de Teorema de Pitágoras y se puede enunciar así:

(Esta parte incluye un recuadro en blanco, para ser completado por los estudiantes.)

La clase comienza con la lectura de la consigna que pide el armado del rompecabezas. Una vez leída, se explica a los alumnos qué les pide la tarea. Luego de esto, los alumnos trabajan en pequeños grupos, completando el armado. Al cabo de un tiempo, se produce una interacción grupal en la que se discuten las partes a, b y c de la actividad. En la ficha que tienen los estudiantes figura también la parte 2). Una vez completadas las partes a, b y c, el EPM3 les dicta lo que pondrán en el recuadro de la parte 2 de la actividad. 
Los estudiantes trabajan recortando las piezas para armar el rompecabezas, haciéndolo de manera bastante autónoma, en pequeños grupos. Una vez terminado esto, se resuelven las partes a) y b) de forma grupal.

A continuación mostramos el episodio en el que se discute grupalmente la parte b). El EPM3 ha realizado la pregunta de la parte 1b) de la actividad, y E3 responde: Esos dos juntos son iguales. Luego se da la siguiente interacción:

\section{Interacción 1 EPM3.}

1 EPM3: ¿Y eso cómo lo expresarían en las áreas? Porque ya armaron el rompecabezas, ya vieron que si ustedes juntaban, o sea, desarmaban los dos cuadrados, y armaban las piezas de alguna forma, les quedaba el cuadrado más grande. Este, ¿no? De mayor área. ¿Se ve eso?

2 Es: Sí.

3 EPM3: Fue lo que hicieron cuando armaron el rompecabezas. Bueno, ¿eso cómo se puede traducir con respecto a las áreas de los cuadrados?

4 E11: Son equivalentes.

5 EPM3: ¿̇as de cuáles? A ver, por ahí ya dijeron algo. Dijeron que una suma, por ahí, no sé, por allá. E3 dijo una suma.

6 E3: La suma de los dos cuadrados es lo mismo que el cuadrado grande.

7 EPM3: ¿Se ve eso? Si ustedes con las 5 fichas (muestra los cinco dedos de la mano) construyen el cuadrado rojo, que es el más grande de todos, el que tiene la mayor área, ¿cómo se traduce eso en términos de áreas? Bueno, que si yo sumo el área de este cuadrado que está acá, capaz que lo hago en este que es más grande, de este cuadrado que está acá (señala), si sumo el área de este cuadrado, con el área de este chiquito amarillo...

8 E12: el área del rojo.

9 EPM3: Me da el área del rojo, ¿no? Que fue lo que hicieron ustedes cuando armaron el puzle. Los dos cuadrados que quedaron construidos eran, estaban superpuestos uno con el otro. O sea, tienen la misma área. Bueno, entonces, esa sería la respuesta. ¿Cómo lo escribirían con sus palabras? Así lo respondemos entre todos a eso.

10 E13: Sumando el área del cuadrado menor.

11 E14: Ponéle letras a los cuadrados.

12 EPM3: Bueno, esa puede ser una opción. Ponerle vértice a los, perdón, ponerle nombre a los vértices de los cuadrados. Pero otra, otra opción podría ser, estos dos cuadrados, están construidos sobre los catetos del triángulo, ¿no?

13 E15: 0 si no, sumando el área de uno de los cuadrados da el área del otro cuadrado, de los otros dos, de los cuadrados menores. 
14 EPM3: Pero, ¿cómo sumas? ¿Un área sola vas a sumar? ¿Cómo sería? Porque vos dijiste: sumando el área de un cuadrado...

15 E15: de los cuadrados menores.

16 EPM3: Bueno, pero, para no decir cuadrados menores, porque, ¿qué querría decir que un cuadrado es menor que otro?

17 E13: Más chico.

18 E14: Más chiquito.

19 EPM3: ¿Qué sería que un cuadrado es más chico que otro? ¿Cómo diríamos?

20 E15: Que tiene menor área.

21 EPM3: Ah, bueno, que el área de uno sería más chica que el área del otro, bueno. Ahora les vuelvo a decir, recuerden que los cuadrados que ustedes recortaron son los cuadrados que están construidos sobre los catetos del triángulo rectángulo. ¿Se ve eso? ¿Cuáles son los catetos de ese triángulo rectángulo? En el triángulo rectángulo tienen nombre los vértices, ¿no? ABC. ¿Cuáles son los catetos de ese triángulo rectángulo?

22 E10: BA, BC y BA.

23 EPM3: BC.

24 E10: y CA.

25 EPM3: Y CA (como aprobando). ¿BA qué es del triángulo?

26 E10: Hipotenusa.

27 EPM3: La hipotenusa. Porque es el lado opuesto al ángulo recto. ¿Se acuerdan?

28 Es: Sí.

29 EPM3: Ya habíamos trabajado eso. Bueno. Entonces, les vuelvo a decir. ¿Cómo podemos escribir eso? A ver.

30 E9: La suma del triángulo amarillo, más el triángulo de colores.

31 EPM3: Está hablando E9. Decí, a ver, el área de

32 E9: El triángulo amarillo

33 EPM3: ¿Triángulo amarillo?

34 Es: Cuadrado.

35 EPM3: Ah,

36 E9: Si le sumás el área del cuadrado de colores.

37 EPM3: A ver si se ve eso que dijo E9. E9 dice: "si sumo el área del cuadrado amarillo con el área del cuadrado ese que tiene varios colores, me da el área de qué cuadrado".

38 Es: Del rojo. 
39 EPM3: Del rojo. Bueno, vamos a escribirlo un poco distinto porque decir cuadrados de colores es como medio...

40 E16: Si se ponen los números del 1 al 5, te da el rojo. Ta.

41 EPM3: ¿Sí? ¿Si sumo los números del 1 al 5?

42 E16: No, si los nombrás.

43 EPM3: Si sumo las áreas.

44 E16: iEso!

45 EPM3: Bueno, eso puede ser otra opción, si sumo las áreas de las figuras numeradas del 1 al 5, podría ser eso, vayan escribiendo, podemos escribirlo de alguna otra manera distinta. (Dicta): Si sumo las áreas.

46 Si sumamos las áreas de las figuras numeradas del 1 al 5, se obtiene el área del cuadrado rojo, por ejemplo.

En la resolución de la parte b) el EPM3 muestra una intención dialógica con los estudiantes, en el sentido de que, por ejemplo, cuando uno de ellos dice son equivalentes, refiriéndose a las áreas de los cuadrados, el docente pregunta clas de cuáles?, con la intención de que el estudiante complete su respuesta (L4 - L6). Sin embargo, podemos establecer varias fases dentro de la interacción que nos muestran elementos correspondientes al patrón extractivo-embudo. Lo esquematizamos a continuación.

L1 - L3: pregunta del EPM3.

L4 - L9: Respuesta de un estudiante (son equivalentes), reacción del EPM3 para que mejore su explicación, enunciado por parte del EPM3.

L9: Reiteración de la pregunta.

L10 - L21: En este segmento, el EPM3 no toma las propuestas de los estudiantes, rechazándolas de diversas maneras, e introduce (en las líneas 12 y 21) sugerencias para que ellos enuncien la propiedad en términos de los cuadrados construidos sobre los catetos y el construido sobre la hipotenusa.

L22 - L 29: Guía hacia la respuesta esperada (va preguntando cuáles son los nombres de los lados del triángulo y vuelve a pedir que establezcan la relación).

L30 - L37: Respuesta divergente de la esperada. Parece tomar la respuesta.

L39 - L 46: Rechazo de la respuesta anterior, nueva propuesta (numerar los cuadrados), y enunciado en estos términos.

Podemos observar, entonces, características propias del patrón extractivo-embudo, aunque no ocurre de forma tan clara como en el caso del EPM1. 
En la línea 9 el EPM3 pide a los alumnos que enuncien esa relación con sus palabras. Los alumnos realizan aportes (L10: Ios cuadrados menores; L11: poner letras a los cuadrados; L30: usando colores para distinguir los cuadrados; L40: numerando los cuadrados). Aunque los estudiantes han generado el enunciado que el EPM3 quiere establecer, el modo en que este pretende que lo hagan genera por momentos sugerencias propias del patrón extractivo-embudo. En efecto, el docente realiza tres intentos (L12, L16, L21-L29) de que los alumnos llamen a los cuadrados involucrados en la tarea cuadrados construidos sobre los catetos y cuadrado construido sobre la hipotenusa. De forma especial entre las líneas 21 y 29, el EPM1 va preguntándoles cuáles de los lados son los catetos del triángulo ABC, y cuál la hipotenusa, para preguntar nuevamente cómo pueden enunciar dicha relación. Sin embargo, en la línea 30, E9 propone usar colores para distinguir los cuadrados, cosa que el EPM3 parece aceptar, pero luego se inclina por la propuesta de numerar la mayoría de los cuadrados. La relación se establece en los siguientes términos: Si sumamos las áreas de las figuras numeradas del 1 al 5, se obtiene el área del cuadrado rojo. Una vez llegados a esta conclusión, para la que los estudiantes tienen adjudicado un significado originado en el rompecabezas que armaron, el EPM3 trabaja con ellos para que enuncien la misma relación usando las medidas de los lados del triángulo: $a, b$ y $c$, que es la parte $1 c$ ) de la actividad. Guiados por sus preguntas, los alumnos van diciendo cuáles son las expresiones de las áreas de cada cuadrado, y finalmente se escribe en el pizarrón: $a^{2}+b^{2}=c^{2}$. El EPM3 les dice que esta relación se conoce como el Teorema de Pitágoras. A partir de eso, el EPM3 dicta el enunciado del teorema.

\section{Interacción 2 EPM3.}

47 EPM3: Les dicto, no he empezado a dictar todavía. Dicto lo que va en ese recuadro entonces. Dice: "en cualquier triángulo rectángulo, es un juego de palabras que a veces es difíil de decir, pero no hay otra forma más sencilla de decirlo, el cuadrado de la medida de la hipotenusa es igual a la suma de los cuadrados de las medidas de los catetos."

48 E8: Es eso, ¿no? (Señala al pizarrón, donde dice $a^{2}+b^{2}=c^{2}$ ).

49 EPM3: Sí, es eso, simbólicamente se escribe así. O sea, en otras palabras, es lo que ustedes estaban diciendo hace un ratito en función de las áreas de esos cuadrados que estaban ahí, ahora eso está dicho en función de los lados del triángulo. O sea, lo que dice, en otras palabras, es, si yo tengo un triángulo rectángulo, y sumo los cuadrados de las medidas de los catetos, eso me da el cuadrado de la medida de la hipotenusa. Es lo que estaban anotando ustedes recién. Bien. 
El enunciado al que llega el EPM3 es el enunciado "oficial actual" del Teorema de Pitágoras (Millán, 2004). Y es, también, el mismo que figura en el texto de donde tomó la actividad (Ochoviet y Olave, 2009). Aunque muchas veces el Teorema de Pitágoras se enuncia en términos de áreas de cuadrados, el enunciado es ambiguo, ya que el cuadrado de un lado de un triángulo podría hacernos pensar en el área de un cuadrado con ese segmento como lado. Pero no hay una referencia explícita a las áreas de los cuadrados en el enunciado del teorema. Sí la hay en la demostración de Euclides, la que realiza en base a las áreas de los cuadrados. Y en los libros de texto, aunque en distinto grado se menciona la interpretación a través de las áreas de los cuadrados, la inmensa mayoría de las aplicaciones que se presentan se refieren al uso del teorema para el cálculo de longitudes. Sin embargo, pensamos que el inicio de la actividad, en la clase del EPM3, donde se pide que los alumnos concluyan el enunciado del teorema a partir de la manipulación de las figuras, introduce un contexto que será el que sostenga, para los estudiantes, el significado establecido para dicho teorema. Los alumnos establecieron el enunciado del Teorema de Pitágoras a partir de la manipulación de las representaciones de las figuras, las que posiblemente tuvieran para ellos un estatus de figuras. Al armar el rompecabezas comprobaron que la relación entre las áreas se verificaba. Luego de enunciada la relación en términos de áreas, a pesar de que el EPM3 establece oralmente que: $O$ sea, en otras palabras, es lo que ustedes estaban diciendo hace un ratito en función de las áreas de esos cuadrados que estaban ahí, ahora eso está dicho en función de los lados del triángulo, no adquiere el mismo significado para los estudiantes. Además, el EPM3 no le dio estatus de teorema al enunciado expresado en términos de las áreas de los cuadrados numerados y del cuadrado rojo, tal vez respetando la actividad tal como fue planteada, o quizá porque vio que los alumnos no daban un enunciado en términos de cuadrados construidos sobre los lados del triángulo rectángulo. El hecho es que el enunciado del teorema como tal lo establece el EPM3, dejando de lado la relación que habían acordado antes, para pasar al contexto de las longitudes. Y además, diciendo que: es un juego de palabras que a veces es difícil de decir, pero no hay otra forma más sencilla de decirlo. Si bien no podemos decir que hay un empobrecimiento de significados, sí se produce un cambio de los significados establecidos en términos de áreas, para pasar al contexto de las longitudes. Y esto lleva a que, en las actividades de aplicación del teorema, el EPM3 se vea obligado a forzar a los estudiantes, que si bien entienden que tienen que usar la herramienta estudiada, pretenden hacerlo utilizando áreas y no longitudes. 
Como ejemplo mostramos parte de la interacción que se produce en la discusión de una de las actividades de aplicación propuestas por el EPM3, que enunciamos a continuación.

Una caña de 30 unidades de largo se apoya verticalmente contra un muro. Si la extremidad superior de la caña se coloca 6 unidades más abajo, ¿en cuántas unidades se desplazará el otro extremo de la caña? (Una caña es un tallo cilíndrico, con nudos macizos y extremos huecos).

Se resuelve de forma grupal. En primer lugar, es discutido el enunciado, y con intervención de los estudiantes se realiza una figura de análisis, a partir de determinar que uno de los catetos del triángulo representado mide 24 unidades.

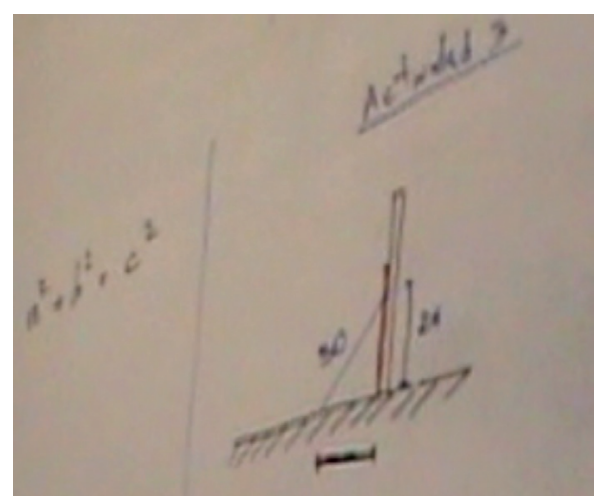

Figura 1. Copia de la figura de análisis realizada en el pizarrón.

Al inicio, un estudiante propone calcular 30 al cuadrado más 24 al cuadrado, idea que el EPM3 toma, entendemos que porque le resulta útil para continuar hacia la resolución del problema. Entonces el EPM3 introduce la necesidad de comprobar si el triángulo es rectángulo. En ese momento E10 propone calcular 30 por 24 dividido 2. Se plantea que hay que aplicar lo que dieron antes. El EPM3 comienza a hacer preguntas en relación a cuáles son los datos, si lo son las longitudes de los catetos (como en la actividad anterior). Finalmente, el EPM3 pregunta: Pero en el Teorema de Pitágoras ¿̇aparece el área del triángulo en algún momento?, a lo que se da la siguiente interacción: 


\section{Interacción 3.1 EPM3.}

1 E3: No, de los cuadrados.

2 EPM3: Aparecen las áreas de los cuadrados que construyo sobre los catetos y sobre la hipotenusa. No aparece el área del triángulo...

(Aquí sigue un diálogo para ver que el área no está bien calculada, no se transcribe porque pensamos que no aporta al análisis que estamos realizando).

\section{E10: La base falta.}

4 EPM3: Entonces no sé, si la base no la tengo, ¿cómo sé el área?

5 E10: Podés poner $x$ por 24.

6 EPM3: Aja, a ver si a esta medida le llamo $x$ por ejemplo. (Escribe $x$ debajo del cateto horizontal). Vuelvo a preguntar, ¿no conocen algún vínculo (señala la igualdad $a^{2}+b^{2}=c^{2}$ ) entre las medidas de los lados de un triángulo rectángulo? Más explícito imposible.

E10 continúa con su idea de hallar el área del triángulo, y corrige la expresión de la misma introduciendo la incógnita $x$. El EPM3 toma como útil esta idea de E10, aunque se ve obligado a sugerir de forma explícita el uso de la relación de Pitágoras. Los estudiantes, sin embargo, insisten en la no existencia de cuadrados en el marco de este problema. Esto nos hace pensar que siguen anclados en el significado original que adjudicaron a los cuadrados de las longitudes de los catetos y de la hipotenusa, como representantes de áreas de cuadrados. Nuevamente el EPM3 necesita explicitar que tendrán que usar la relación, y directamente les pregunta cómo sería plantear dicha igualdad en este caso, configurando así el patrón extractivo-embudo.

\section{Interacción 3.2 EPM3.}

\section{E11: No hay cuadrados.}

8 EPM3: No hay cuadrados dibujados, es cierto. Pero las medidas de los lados las tengo. De algunos, por lo menos.

9 E11: Claro, pero...

10 EPM3: ¿Cómo sería plantear esta igualdad (señala $a^{2}+b^{2}=c^{2}$ ) en este triángulo (señala el triángulo)? ¿Cómo sería? 
Una vez llegados a este punto, el problema se termina de resolver grupalmente.

En síntesis, creemos que el pasaje del contexto de las áreas al de las longitudes, al momento de enunciar el Teorema de Pitágoras, resultó falto de significado para los estudiantes. Incluso, el enunciado del teorema en términos de las áreas de los cuadrados numerados que dan los alumnos, no recibe de parte del EPM3 el título de Teorema de Pitágoras. Entendemos que el futuro docente se vio obligado a enunciar el teorema en los términos en que la tradición escolar lo señala y en los que se utiliza para aplicaciones, pero esto distanció dicho enunciado del que los alumnos habían generado, y para el que tenían construido un sentido. Entre un contexto y otro solo medió una frase del EPM3: O sea, en otras palabras, es lo que ustedes estaban diciendo hace un ratito en función de las áreas de esos cuadrados que estaban ahí, ahora eso está dicho en función de los lados del triángulo. Creemos que esto condiciona los siguientes episodios, en el sentido de promover la configuración de los patrones extractivo-embudo, disminuyendo notoriamente la intención dialógica del EPM3, así como la riqueza en la negociación de significados.

\section{DISCUSIÓN}

En los episodios de los dos EPM que aquí se han analizado observamos la configuración del patrón extractivo-embudo, de modo más claro para el EPM1 que para el EPM3. Como plantean Bauersfeld et al. (1985: 9):

A menudo el juego de preguntas y respuestas en las clases de matemática acontece bajo la pretensión de un discurso abierto, liberal, en el que los estudiantes participan activamente. En oposición a esto, los microanálisis señalan estructuras ocultas y estereotipadas, y prácticas estructurantes del discurso de todos los días en la clase de matemática. Estas estructuras y prácticas estructurantes sostienen el funcionamiento fluido de la interacción, no son conscientemente percibidos por estudiantes y docentes, y producen efectos no deseados en los resultados de aprendizaje de los estudiantes y en sus hábitos de aprendizaje (traducción de los autores).

En estos episodios, particularmente, las interacciones desarrolladas presentan la apariencia de un diálogo abierto en el que cada EPM realiza preguntas a sus alumnos, y estos responden. Sin embargo, analizados con mayor profundidad, 
con el auxilio del protocolo de observación (Tabla 4), surgen las estructuras estereotipadas que acabamos de señalar, y que conducen a la culminación de la tarea propuesta, pero a costa de la no consideración de varias intervenciones de estudiantes, así como de la orientación de las mismas por parte de cada EPM.

Al analizar estos episodios hemos observado lo que motiva este artículo, es decir, la configuración del patrón extractivo-embudo asociada a la evocación o la institucionalización, en la clase, de determinado concepto matemático. En el caso del EPM1, pensamos que, además de que la respuesta el valor de x cuyo correspondiente es el cero es considerada más útil por él, en el sentido de permitir determinar la raíz de la función dada; también es considerada la definición matemática de raíz de una función, en tanto que la abscisa del punto de corte de la representación gráfica de la función con el eje x (respuesta dada por E6), se entiende como una interpretación geométrica de este concepto. Y en el caso del EPM3, en la clase se había negociado un significado para el Teorema de Pitágoras, sostenido por el contexto de la actividad del rompecabezas, en base a las medidas de las superficies de los cuadrados, y se pierde en el enunciado del teorema en términos de longitudes. Además de esto, los nombres que los estudiantes adjudican a las figuras involucradas ("las figuras numeradas del 1 al 5", "el cuadrado rojo") y que utilizan para su enunciado del teorema, son abandonados por el EPM3 al momento de dar estatus matemático al enunciado del teorema.

\section{CONCLUSIONES}

En este trabajo hemos considerado algunos episodios de interacción de clases de estudiantes de profesorado de matemática de Uruguay, cuando realizan el último curso de su práctica docente. En particular se han analizado aquellos donde el objetivo de la interacción consistía en conseguir que los alumnos enunciaran una definición o una proposición (la definición de raíz de una función, y el Teorema de Pitágoras, respectivamente). En ambos casos se configura el patrón extractivo-embudo, y la definición o la proposición, en la forma en que cada EPM quiere que sean enunciados, son explicitados por los propios EPM, alejándose de las intervenciones y aportes de sus alumnos. Según Voigt (1995), las evidencias nos muestran que los docentes tienen dificultades para tomar las intervenciones de sus alumnos cuando estas difieren de las respuestas esperadas por ellos. Agregamos que este fenómeno se torna más reiterado cuando se 
trata de establecer un concepto o proposición matemático preciso, como el caso de la definición de raíz de una función o el enunciado del Teorema de Pitágoras. Los dichos de los EPM muestran que sienten el mandato de reproducir de manera precisa los enunciados de las matemáticas, tal como aparecen en los libros o en sus apuntes de las clases de profesorado. De esta forma, los EPM presentan las matemáticas como un producto construido externamente, dejando de lado los procesos que podrían llevar adelante los estudiantes para construir dicho conocimiento. Esta concepción es ampliamente extendida en torno a las matemáticas que, en efecto, son generalmente consideradas la ciencia por excelencia, una disciplina uniforme (Steinbring, 2005). Esta idea también influye la concepción sobre la enseñanza de las matemáticas que mucha gente tiene.

Steinbring (2005: 15), citando a Freudenthal (1973: 114):

Es cierto que palabras como matemática, lenguaje, y arte tienen un doble significado. En el caso del arte es obvio. Existe un arte terminado que es el que estudia el historiador del arte, y existe un arte ejercitado por el artista... Cada matemático sabe al menos inconscientemente que al lado de las matemáticas ya hechas existen las matemáticas como una actividad. Pero este hecho casi nunca se señala, y no todos los no matemáticos son conscientes de ello (traducción de los autores).

Esta forma de ver las matemáticas como actividad lleva a pensar en su aprendizaje como un proceso activo de construcción de conocimiento.

La concepción sostenida por los EPM, que en definitiva considera las matemáticas como ya construidas o existentes, los Ileva a forzar la situación, llegado el momento de establecer una definición, propiedad o teorema, al punto de dejar de lado los aportes de los alumnos, y dar ellos mismos el enunciado, lo cual afecta la interacción y los significados que los estudiantes construyen.

Consideramos que uno de los aportes de este trabajo al campo de la Formación del Profesor de Matemáticas consiste en poner en evidencia lo señalado anteriormente: la relación existente entre la configuración del patrón extractivo-embudo y el establecimiento en la clase de un resultado matemático. Los patrones de interacción se configuran a través de la rutina y, por tanto, son, en general, inconscientes. La evidencia de que, al momento de querer establecer en clase determinados resultados de las matemáticas, nos encontraremos con el pensamiento de los estudiantes, apegado al contexto y experiencias que han tenido anteriormente, es de suma importancia en la formación docente. Pudimos ver, para el caso del EPM3, que promovía interacciones dialógicas con sus 
alumnos que, en el caso de institucionalizar el Teorema de Pitágoras en su versión oficial, o de plantear una situación donde debe aplicarse en un contexto distinto de aquel en que fue introducido, termina configurando el patrón de interacción extractivo-embudo.

A partir de lo anterior, creemos que este trabajo proporciona una herramienta para el análisis de clases de matemáticas, usando transcripciones de episodios seleccionados con ese fin. Si bien esta herramienta se focaliza en algunos aspectos de la clase y por eso resulta parcial, consideramos que, para los futuros profesores y docentes en ejercicio de su profesión, puede ser una forma de hacerse conscientes de ciertas rutinas que, de diferentes formas, no consideran, parcial o totalmente, el pensamiento de los estudiantes.

Pensamos que sería muy importante tratar estas cuestiones en los cursos de formación de profesores, ya que los patrones de interacción como el extractivo o el embudo son generalmente inconscientes y se van estableciendo de forma interactiva. El abordaje de los mismos permitirá la consideración de alternativas, tanto en relación al análisis didáctico de las planificaciones de clase como en las propias interacciones, que redunden en la construcción de significados matemáticos más ricos durante las clases, lo mismo las de la práctica docente que las clases que los futuros profesores impartan durante el ejercicio de su profesión.

\section{REFERENCIAS}

Bauersfeld, H., Krummheuer, G. y Voigt, J. (1985). Interactional Theory of Learning and Teaching Mathematics and Related Microetnographical Studies. En H. G Steiner, Proceedings of the TME 1985. Bielefeld: IDM.

Blanco, L. J. (1996). Aprender a enseñar Matemáticas. Tipos de conocimientos. En J. Giménez, S. Llinares y M. V. Sánchez (eds.), El proceso de llegar a ser un profesor de primaria. Cuestiones desde la educación matemática, 199-221. Granada: Comares.

Godino, J. D. y Llinares, S. (2000). El interaccionismo simbólico en educación matemática. Educación Matemática. 12 (1). 70-92.

Homilka, L (2008). Influencia de las prácticas docentes en la visión de estudiantes y profesores de matemática acerca de la matemática en el aula y las decisiones didácticas. Tesis de maestría no publicada. CICATA-IPN.

Millán, A. (2004). Euclides. La fuerza del razonamiento matemático. Tres Cantos: Nivola. Ochoviet, C. y Olave, M. (2009). Matemática 3. Montevideo: Santillana. 
Olave, M. (2013). Modelos de profesores formadores de matemáticas: ¿Cuáles son y en qué medida se transmiten a los futuros docentes? Un estudio de caso. (Tesis de doctorado no publicada). CICATA, del IPN, México. Disponible en http://www.matedu. cicata.ipn.mx/tesis/doctorado/olave_2013.pdf.

Ponte, J. y Chapman, O. (2006). Mathematics Teachers' Knowledge and Practices. In A. Gutierrez y P. Boero (eds.), Handbook of Research on the Psychology of Mathematics Education: Past, Present and Future (pp. 461-494). Roterdham: Sense.

Radford, L. (2010). Book Review: Classroom Interaction: Why is it Good, Really? En B. Schwarz, T. Dreyfus y R. Hershkowitz (eds.) (2009). Transformation of Knowledge through Classroom Interaction. Oxon: Routledge.

Sierpinska, A. y Lerman, S. (1996). Epistemologies of Mathematics and of Mathematics Education. En A. J. Bishop, M. A. (Ken) Clements, C. Keitel, J. Kilpatrick y C. Laborde (eds.). International Handbook of Mathematics Education, 1, 827- 876. Dordrecht, HL: Kluwer, A. P.

Steinbring, H. (2005). The Construction of New Mathematical Knowledge in Classroom Interaction-An Epistemological Perspective. Berlin: Springer.

Stephan, M. y Cobb, P. (2003). The Methodological Approach to Classroom-based Research. En Stephan, M., Bowers, J y Cobb. P (eds). Supporting Students' Development of Measuring Conceptions: Analyzing Students' Learning in Social Context. Journal for Research in Mathematics Education Monograph № 12 (pp. 36-50). Reston, VA: National Council of Teachers of Mathematics.

Voigt, J. (1985). Patterns and Routines in Classroom Interactions. Recherches en Didactique des Mathématiques. 6 (1), 69-118.

Voigt, J. (1995). Thematic Patterns of Interaction and Sociomathematical Norms. En H. Bauersfeld y P. Cobb (eds.), The Emergence of Mathematical Meaning: Interaction in Classroom Cultures. Hillsdale, NJ: Lawrence Erlbaum Associates.

Wood, T. (1994). Patterns of Interaction and the culture of Mathematics Classrooms. Cultural Perspectives on the Mathematics Classrooms. Mathematics Education Library, 14, 149-168.

Wood, T. (1998). Alternative Patterns of Communication in Mathematics Classes: Funneling or Focusing? En H. Steinbring, M. Bartolini y A. Sierpinska (eds.), Language and Communication in the Mathematics Classroom. Boston: The National Council of Teachers of Mathematics, Inc. 